\title{
Cystic fibrosis newborn screening programs: implications of the CFTR variant spectrum in nonwhite patients
}

\author{
Lynn Pique, BS1, Steve Graham, PhD², Michelle Pearl, PhD³ , Martin Kharrazi, $\mathrm{PhD}^{4}$ and Iris Schrijver, $\mathrm{MD}^{1,5}$
}

Purpose: Cystic fibrosis newborn screening (CFNBS) has been offered across the United States since 2010. However, as compared with white patients with $C F, C F T R$ variant identification in nonwhite populations remains inequitable. Utilizing the recent characterization of the nonwhite CF variant spectrum, we examined the effectiveness of current CFNBS molecular panels in identifying affected nonwhite newborns.

Methods: Based on a cross-sectional evaluation of genotyping data from the CF Foundation Patient Registry that compared 3,496 nonwhite with 22,206 white CF patients, the current CFNBS algorithms used in the 50 states and the District of Columbia were analyzed. We assessed the percentage of CF patients of Hispanic, African, Asian, and Native American heritage who would not be identified by the molecular panels most commonly used.
Results: Compared with whites, variant detection was significantly lower in Hispanic, black, and Asian newborns ( $P \leq 0.0001$ each), as well as in Native American newborns ( $P$ values ranged from 0.001 to 0.0003), for the most common CFNBS panels.

Conclusion: This study provides a perspective on the applicability of current panels to a diverse population and enables CFNBS programs to consider more inclusive test approaches to facilitate diagnosis, timely clinical intervention, and enhanced prognosis for CF patients of nonwhite and mixed ethnicities.

Genet Med advance online publication 5 May 2016

Key Words: CFTR; cystic fibrosis; mutation detection; newborn screening; nonwhite

\section{INTRODUCTION}

Cystic fibrosis (CF; OMIM 219700) is an autosomal recessive condition caused by mutations in the CFTR gene (OMIM ${ }^{*} 602421$ ) that disrupt the production of the cystic fibrosis transmembrane conductance regulator (CFTR), a protein critical for chloride ion channel formation in the exocrine epithelial cells of multiple tissues and organs. A hallmark of classic CF, and the major contributor to morbidity and mortality, is pulmonary disease, which manifests as chronic respiratory inflammation and infection. ${ }^{1}$ Additional clinical features include failure to thrive, pancreatic insufficiency, intestinal obstruction, and infertility. The birth prevalence of CF in the United States approximates 1:3,500 overall, but Ashkenazi Jews and nonHispanic whites are most frequently affected, with respective prevalences of $\sim 1: 2,270$ and up to 1:2,500 individuals. ${ }^{2-5} \mathrm{CF}$ is reported in $\sim 1: 15,000$ blacks, 1:35,000 Asians, and 1:10,900 Native Americans. ${ }^{5,6}$

Clinically, CF can be established by the presence of at least one distinctive phenotypic feature as well as laboratory confirmation of functional CFTR abnormality, primarily by verification of an elevated sweat chloride $\left(\mathrm{Cl}^{-}\right)$concentration and/or the identification of a pathogenic sequence change in each of the two copies of the CFTR gene. ${ }^{7}$ Early recognition and diagnosis can be challenging, however, because presenting symptoms are not specific to $\mathrm{CF} .{ }^{8}$ Consequently, a CF diagnosis predicated on the manifestation of clinical symptoms (excluding newborns diagnosed with meconium ileus or with a positive family history) is delayed, on average, until well into the second year of life, which commonly results in compromised clinical status as compared with early diagnosis and intervention. ${ }^{9-11}$ In an effort to minimize the potential for diagnostic delay, CF newborn screening (NBS) programs were implemented across the United States, in all 50 states and the District of Columbia, by $2010 .^{12}$ The median age of CF diagnosis has since been advanced to $\sim 2-4$ weeks of life. ${ }^{13}$

Although CF newborn screening (CFNBS) methodologies vary between programs, the first step always involves the measurement of immunoreactive trypsinogen (IRT) in dried blood spots. When an elevated IRT level is detected, secondary testing consisting of a repeat IRT, DNA testing (most often comprising a mutation panel), or a combination of the two is performed. Newborns with a second occurrence of elevated IRT and/or a single identified CFTR variant are usually considered screening test-positive and may receive more extensive molecular analysis or are referred for clinical evaluation and sweat chloride measurement. The CFTR variants chosen for the initial DNA test affect the clinical sensitivity of NBS algorithms. Because the distribution and frequency of CFTR sequence changes vary with respect to specific ethnic groups and geographic locations, ${ }^{5,14,15}$ panel composition is an important consideration for

\footnotetext{
${ }^{1}$ Department of Pathology, Stanford University Medical Center, Stanford, California, USA; ${ }^{2}$ Genetic Disease Screening Program, California Department of Public Health, Richmond, California, USA; ${ }^{3}$ Sequoia Foundation, La Jolla, California, USA; ${ }^{4}$ Environmental Health Investigations Branch, California Department of Public Health, Richmond, California, USA; ${ }^{5}$ Department of Pediatrics, Stanford University Medical Center, Stanford, California, USA. Correspondence: Iris Schrijver (irismd@stanford.edu)

Submitted 21 November 2015; accepted 8 March 2016; advance online publication 5 May 2016. doi:10.1038/gim.2016.48
} 
NBS programs, particularly in the genetically diverse and ethnically admixed population of the United States.

We recently published a cross-sectional analysis comparing 3,496 nonwhite patients to 22,206 white CF patients using the genotyping data of patients from all ethnicities in the CF Foundation Patient Registry. ${ }^{16}$ In that study, the spectrum and allele frequencies of CFTR sequence variants in nonwhite CF patients were categorized by ancestry and the most frequent sequence changes were tabulated. ${ }^{16}$ The objective of the present study, in contrast, was twofold: first, to determine which algorithms and molecular analyses are currently used for CFNBS in the United States and, second, to utilize our characterization of the nonwhite CF variant spectrum to analyze the impact of different panels on the detection of CFTR variants in nonwhite CF patients as compared with whites.

\section{MATERIALS AND METHODS}

\section{CFTR genotyping and CF Foundation Patient Registry data} analysis

The spectrum of genotypes in non-Hispanic whites and Ashkenazi Jews is well characterized because these populations are the most commonly affected with $\mathrm{CF}^{3-5}$ To more completely elucidate the spectrum and frequencies of CFTR variants in affected US minorities, and to facilitate early identification in these populations, we combined our genotyping results from nonwhite patients with genotype data from the CF Foundation Patient Registry to perform a cross-sectional analysis. ${ }^{16}$ Nonwhite CF patients with incomplete CFTR genotypes (0 or 1 previously identified CFTR variants) as well as those who had no prior genotype testing who were enrolled in the CF Foundation Patient Registry through 161 US CF centers were eligible for genotyping in our study. Nonwhite patients, for the purposes of this study, were individuals who self-identified as black, Asian, Native American, East Indian, or Middle Eastern. White and Hispanic CF patients were excluded from the genotyping. CF patients of more than one race/ethnicity were included for genotyping unless they reported solely white and Hispanic ancestry. CFTR variants established to be benign were excluded. This assessment was performed by the following combined approaches: (i) reported variant allele frequency (>1\%); (ii) information in public databases, such as dbSNP (http://www.ncbi.nlm.nih.gov/projects/SNP/); (iii) frequency of recurrence observed in our internal clinical diagnostic database; and (iv) reports in the literature regarding functional studies or segregation with disease. Sequence changes of uncertain clinical significance were included but not further evaluated to assess the degree of pathogenicity.

Genotyping was performed by the clinical diagnostic Stanford Molecular Pathology laboratory as previously described. ${ }^{16}$ Leftover DNA samples with only one or no variant(s) found by sequencing were de-identified and assayed separately for CFTR copy-number variants by multiplex ligation-dependent probe amplification on a research basis. Breakpoints of identified rearrangements were also characterized. A total of 140 CF patients were enrolled and genotyped over the course of the study period, and their clinical diagnostic test results were appended to their CF Foundation Patient Registry entries by CF center staff. Thus, entries in the CF Foundation Patient Registry were enriched by the study. Using the genotyping data of patients from all ethnicities from the CF Foundation Patient Registry, a cross-sectional analysis comparing 3,496 nonwhite patients with 22,206 white CF patients was performed, and the spectrum of CFTR sequence variants and their allelic frequencies in nonwhite CF patients were characterized. ${ }^{16}$

\section{Survey of state NBS programs and DNA testing comparisons}

For the four molecular test approaches most widely implemented by CFNBS programs in the United States (Table 1), we assessed the number of current CF patients across ethnic ancestry groups in the 2013 CF Foundation Patient Registry who would not be identified by the given testing algorithm. For this study, we defined "current CF patients" as those who were alive and seen at a CF center within the past year of the data set. Patients with self-declared Hispanic-only ancestry were assigned to the "Hispanic Only" category. Nonwhite patients with any African, Asian, or Native American ancestry were categorized in the "Black-Any," "Asian-Any," and "Native-Any" groups, respectively. The CF Foundation Patient Registry database was analyzed using SAS statistical software (version 12.1; SAS Institute, Cary, NC), and, per ethnic group, the frequencies were determined for the number of sequence variants identified by each DNA testing method. Chi-squared statistics were performed and $P$ values were calculated using "White Only" as the reference group to establish whether observed distribution differences between ethnic groups in the number of CFTR variants identified by each molecular test were statistically significant. Additional chi-squared analyses were completed to determine the statistical significance of observed differences between testing platforms in the percentages of each ethnic group for whom zero or two mutations would be detected. State CFNBS programs were contacted via telephone and e-mail regarding their CF screening methodologies. Each state's 2013 birth data $^{17}$ and details of their newborn CF screening, particularly in regard to DNA testing, were compiled in a list of DNA testing methods/ panels (Table 2).

\section{RESULTS}

All 50 states and the District of Columbia were surveyed regarding their CFNBS testing algorithms and, when applicable, the details of their molecular test methods (Table 2 and Figure 1). Every program begins by measuring newborn IRT levels. An elevated IRT reading is followed by a repeat IRT measurement and/or DNA testing. Eight programs $(8 / 51=15.7 \%)$ do not currently include DNA testing as part of their CFNBS; in these circumstances, two elevated IRT levels comprise a positive CFNBS result. The remaining programs $(43 / 51=84.3 \%)$, however, incorporate either one or two tiers of DNA testing into their algorithms. Three commercially available panels represent the most commonly implemented approaches: the InPlex CF 
Table 1 Variants included in the molecular testing panels most frequently used by cystic fibrosis newborn screening programs in the United States

\begin{tabular}{|c|c|c|c|c|}
\hline Variants $^{\mathrm{a}}$ & $\begin{array}{c}\text { p.Phe } 508 \text { del } \\
(N=1 \text { variant })\end{array}$ & $\begin{array}{c}\text { Hologic InPlex CF molecular } \\
\text { test }^{\mathrm{b}}(N=23 \text { variants })\end{array}$ & $\begin{array}{l}\text { Luminex xTAG CF39 kit v2 } \\
\text { ( } N=39 \text { variants })\end{array}$ & $\begin{array}{l}\text { Hologic CF InPlex card } \\
\quad(N=41 \text { variants })\end{array}$ \\
\hline p.F508del & $\bullet$ & $\bullet$ & $\bullet$ & $\bullet$ \\
\hline p.A455E & & $\bullet$ & $\bullet$ & $\bullet$ \\
\hline p. G85E & & $\bullet$ & $\bullet$ & $\bullet$ \\
\hline p. G542X & & $\bullet$ & $\bullet$ & $\bullet$ \\
\hline p.G551D & & $\bullet$ & $\bullet$ & $\bullet$ \\
\hline p.1507del & & $\bullet$ & $\bullet$ & $\bullet$ \\
\hline p.N1303K & & - & $\bullet$ & $\bullet$ \\
\hline p.R117H & & $\bullet$ & $\bullet$ & $\bullet$ \\
\hline p.R334W & & $\bullet$ & $\bullet$ & $\bullet$ \\
\hline p.R347P & & $\bullet$ & $\bullet$ & $\bullet$ \\
\hline p. $R 553 X$ & & $\bullet$ & $\bullet$ & $\bullet$ \\
\hline p.R560T & & $\bullet$ & $\bullet$ & $\bullet$ \\
\hline p.R1162X & & $\bullet$ & $\bullet$ & $\bullet$ \\
\hline p.W1282X & & $\bullet$ & $\bullet$ & $\bullet$ \\
\hline $621+1 G>T$ & & $\bullet$ & $\bullet$ & $\bullet$ \\
\hline $711+1 G>T$ & & $\bullet$ & $\bullet$ & $\bullet$ \\
\hline $1717-1 G>A$ & & $\bullet$ & $\bullet$ & $\bullet$ \\
\hline $1898+1 G>A$ & & $\bullet$ & $\bullet$ & $\bullet$ \\
\hline 2184delA & & $\bullet$ & $\bullet$ & $\bullet$ \\
\hline $2789+5 G>A$ & & $\bullet$ & $\bullet$ & $\bullet$ \\
\hline $3120+1 G>A$ & & $\bullet$ & $\bullet$ & $\bullet$ \\
\hline 3659de/C & & $\bullet$ & $\bullet$ & $\bullet$ \\
\hline $3849+10 k b C>T$ & & - & - & $\bullet$ \\
\hline p.A559T & & & $\bullet$ & \\
\hline p.D1152H & & & & $\bullet$ \\
\hline p.D1270N & & & & $\bullet$ \\
\hline p.E60X & & & & $\bullet$ \\
\hline p.F508C & & * & $\bullet$ & $\bullet$ \\
\hline p.I148T & & & & $\bullet$ \\
\hline p.I506V & & & $\bullet$ & \\
\hline p.I507V & & & $\bullet$ & \\
\hline p.M1101K & & & $\bullet$ & \\
\hline p.Q493X & & & & $\bullet$ \\
\hline p.R347H & & & $\bullet$ & $\bullet$ \\
\hline p.S549N & & & $\bullet$ & $\bullet$ \\
\hline p.S549R & & & $\bullet$ & \\
\hline p.S549R $(A>C)$ & & & & $\bullet$ \\
\hline p.S549R (T>G) & & & & $\bullet$ \\
\hline p.S1255X & & & $\bullet$ & \\
\hline p.V520F & & & $\bullet$ & $\bullet$ \\
\hline p.Y122X & & & $\bullet$ & $\bullet$ \\
\hline p.Y1092X & & & - & \\
\hline p.Y1092X $(C>A)$ & & & & $\bullet$ \\
\hline p.Y1092X (C>G) & & & & $\bullet$ \\
\hline 394delTT & & & $\bullet$ & $\bullet$ \\
\hline 1078delT & & & $\bullet$ & $\bullet$ \\
\hline $1898+5 G>T$ & & & $\bullet$ & \\
\hline $2183 A A>G$ & & $*$ & $\bullet$ & $\bullet$ \\
\hline 2307insA & & & $\bullet$ & \\
\hline $3849+4 G>A$ & & & & $\bullet$ \\
\hline 3876delA & & & $\bullet$ & $\bullet$ \\
\hline 3905insT & & & $\bullet$ & $\bullet$ \\
\hline $5 / 7 / 9 \mathrm{~T}^{\mathrm{c}}$ & & $\bullet$ & $\bullet$ & $\bullet$ \\
\hline
\end{tabular}

avariants are listed according to legacy nomenclature. ACMG 23 Carrier Screening Panel variants are shown in italics. The p.F508C, p.I507V, and p.I506V benign variants and the p.1148T polymorphism are shown in bold. 'Variants used for analytical interpretation only are indicated by an asterisk. "Polypyrimidine tract variants in intron 8 (IVS8; intron 9, IVS9 in sequential nomenclature). 
Table 2 Cystic fibrosis newborn screening programs in the United States (as of 25 August 2015)

\begin{tabular}{|c|c|c|c|c|c|c|}
\hline Program & $\begin{array}{l}\text { No. of } \\
\text { births, 2013 } \\
\text { (ref. 21) }\end{array}$ & $\begin{array}{l}\text { Percentage of } \\
\text { total US births, } \\
2013 \text { (ref. 21) }\end{array}$ & $\begin{array}{l}\text { Testing } \\
\text { algorithm }\end{array}$ & DNA testing method & $\begin{array}{l}\text { No. of } \\
\text { variants } \\
\text { tested }^{\mathrm{a}}\end{array}$ & 5/7/9T testing ${ }^{b}$ \\
\hline Alabama & 58,167 & $1.5 \%$ & IRT/DNA & Hologic CF InPlex card & 40 & Reflex test, reported with $\mathrm{p} . \mathrm{R} 117 \mathrm{H}$ \\
\hline Alaska & 11,446 & $0.3 \%$ & IRT/IRT & $\mathrm{N} / \mathrm{A}$ & $\mathrm{N} / \mathrm{A}$ & $\mathrm{N} / \mathrm{A}$ \\
\hline Arizona & 85,600 & $2.2 \%$ & IRT/DNA & Hologic CF InPlex card & 41 & Always reported \\
\hline Arkansas & 37,832 & $1.0 \%$ & IRT/DNA & Hologic CF InPlex card & 41 & Reflex test, reported with p.R117H \\
\hline California & 494,705 & $12.6 \%$ & $\begin{array}{l}\text { IRT/DNA/ } \\
\text { DNA }\end{array}$ & $\begin{array}{l}\text { Modified Luminex/full CFTR } \\
\text { sequencing }\end{array}$ & $\begin{array}{l}40 / \text { full } \\
\text { sequencing }\end{array}$ & $\mathrm{N} / \mathrm{A}$ \\
\hline Colorado & 65,007 & $1.7 \%$ & IRT/IRT/DNA & Luminex xTAG CF39 kit v2 & 39 & Reflex test, reported with p.R117H \\
\hline Connecticut & 36,085 & $0.9 \%$ & IRT/DNA & Hologic CF InPlex card & 40 & Reported to CF center only \\
\hline Delaware & 10,831 & $0.3 \%$ & IRT/IRT/DNA & Hologic CF InPlex card & 40 & Reflex test, reported with $\mathrm{p} . \mathrm{R} 117 \mathrm{H}$ \\
\hline District of Columbia & 9,288 & $0.2 \%$ & IRT/DNA & Luminex xTAG CF39 kit v2 & 39 & Reflex test, reported with p.R117H \\
\hline Florida & 215,407 & $5.5 \%$ & IRT/DNA & Hologic CF InPlex card & 40 & Reflex test, reported with p.R117H \\
\hline Georgia & 128,748 & $3.3 \%$ & IRT/DNA & Hologic InPlex CF molecular test & 23 & Reflex test, reported with p.R117H \\
\hline Hawaii & 18,987 & $0.5 \%$ & $\begin{array}{l}\text { IRT/IRT/DNA } \\
\text { DNA }\end{array}$ & $\begin{array}{l}\text { Integrated Genetics Cfplus/full } \\
\text { CFTR sequencing }\end{array}$ & $\begin{array}{l}\text { 97/full } \\
\text { sequencing }\end{array}$ & $\mathrm{N} / \mathrm{A}$ \\
\hline Idaho & 22,383 & $0.6 \%$ & IRT/IRT & N/A & N/A & N/A \\
\hline Illinois & 156,931 & $4.0 \%$ & IRT/DNA & Hologic CF InPlex card & 41 & Reflex test, reported with p.R117H \\
\hline Indiana & 83,102 & $2.1 \%$ & IRT/DNA & Hologic CF InPlex card & 40 & Reflex test, reported with p.R117H \\
\hline lowa & 39,094 & $1.0 \%$ & IRT/DNA & Hologic CF InPlex card & 40 & Reflex test, reported with p.R117H \\
\hline Kansas & 38,839 & $1.0 \%$ & IRT/DNA & Hologic CF InPlex card & 40 & Reflex test, reported with p.R117H \\
\hline Kentucky & 55,686 & $1.4 \%$ & IRT/DNA & Hologic InPlex CF molecular test & 23 & Reflex test, reported with p.R117H \\
\hline Louisiana & 63,201 & $1.6 \%$ & IRT/DNA & Luminex xTAG CF39 kit v2 & 39 & Always reported \\
\hline Maine & 12,776 & $0.3 \%$ & IRT/DNA & Luminex xTAG CF39 kit v2 & 39 & Reflex test, reported with p.R117H \\
\hline Maryland & 71,953 & $1.8 \%$ & IRT/IRT & N/A & N/A & $N / A$ \\
\hline Massachusetts & 71,788 & $1.8 \%$ & IRT/DNA & Luminex xTAG CF39 kit v2 & 39 & Reflex test, reported with p.R117H \\
\hline Michigan & 113,489 & $2.9 \%$ & IRT/DNA & Hologic CF InPlex card & 40 & Reflex test, reported with p.R117H \\
\hline Minnesota & 69,159 & $1.8 \%$ & IRT/DNA & Luminex xTAG CF39 kit v2 & 39 & Reflex test, reported with p.R117H \\
\hline Mississippi & 38,634 & $1.0 \%$ & IRT/DNA & Luminex xTAG CF39 kit v2 & 39 & Reflex test, reported with p.R117H \\
\hline Missouri & 75,296 & $1.9 \%$ & IRT/DNA & Hologic CF InPlex card & 40 & Reflex test, reported with p.R117H \\
\hline Montana & 12,377 & $0.3 \%$ & $\begin{array}{l}\text { IRT/IRT/ } \\
\text { DNA }^{d}\end{array}$ & Hologic InPlex CF molecular test & 23 & Not reported \\
\hline Nebraska & 26,095 & $0.7 \%$ & $\begin{array}{l}\text { IRT/IRT/DNA } \\
\text { DNA }\end{array}$ & $\begin{array}{l}\text { p.Phe } 508 \text { del/ Luminex xTAG } \\
\text { CF39 kit v2 }\end{array}$ & $1 / 39$ & Reflex test, reported with p.R117H \\
\hline Nevada & 35,030 & $0.9 \%$ & IRT/IRT & N/A & N/A & N/A \\
\hline New Hampshire & 12,396 & $0.3 \%$ & IRT/DNA & Luminex xTAG CF39 kit v2 & 39 & Reflex test, reported with p.R117H \\
\hline New Jersey & 102,575 & $2.6 \%$ & IRT/IRT/DNA & p.Phe508del & 1 & N/A \\
\hline New Mexico & 26,354 & $0.7 \%$ & IRT/IRT & N/A & N/A & $\mathrm{N} / \mathrm{A}$ \\
\hline New York & 236,980 & $6.0 \%$ & IRT/DNA & Hologic CF InPlex card & 40 & Reflex test, reported with p.R117H \\
\hline North Carolina & 119,002 & $3.0 \%$ & IRT/DNA & Hologic CF InPlex card & 41 & Reflex test, reported with p.R117H \\
\hline North Dakota & 10,599 & $0.3 \%$ & IRT/DNA & Hologic CF InPlex card & 40 & Reflex test, reported with p.R117 $\mathrm{H}^{c}$ \\
\hline Ohio & 138,936 & $3.5 \%$ & IRT/DNA & Hologic CF InPlex card & 40 & Reflex test, reported with p.R117H \\
\hline Oklahoma & 53,369 & $1.4 \%$ & IRT/DNA & Luminex xTAG CF39 kit v2 & 39 & Reflex test, reported with p.R117H \\
\hline Oregon & 45,155 & $1.1 \%$ & IRT/IRT & N/A & $\mathrm{N} / \mathrm{A}$ & $\mathrm{N} / \mathrm{A}$ \\
\hline Pennsylvania & 140,921 & $3.6 \%$ & IRT/DNA & Luminex xTAG CF39 kit v2 & 39 & Reflex test, reported with p.R117H \\
\hline Rhode Island & 10,809 & $0.3 \%$ & IRT/DNA & Luminex xTAG CF39 kit v2 & 39 & Reflex test, reported with p.R117H \\
\hline South Carolina & 56,795 & $1.4 \%$ & IRT/IRT & N/A & $\mathrm{N} / \mathrm{A}$ & N/A \\
\hline South Dakota & 12,248 & $0.3 \%$ & IRT/DNA & Hologic CF InPlex card & 40 & Reflex test, reported with p. $\mathrm{R} 117 \mathrm{H}^{c}$ \\
\hline Tennessee & 79,992 & $2.0 \%$ & IRT/IRT & N/A & N/A & N/A \\
\hline Texas & 387,340 & $9.9 \%$ & IRT/IRT/DNA & Hologic CF InPlex card & 40 & Reflex test, reported with p.R117H \\
\hline Utah & 50,957 & $1.3 \%$ & IRT/IRT/DNA & Ambry Genetics & 32 & $\mathrm{~N} / \mathrm{A}$ \\
\hline Vermont & 5,975 & $0.2 \%$ & IRT/DNA & Luminex xTAG CF39 kit v2 & 39 & Reflex test, reported with p.R117H \\
\hline Virginia & 102,147 & $2.6 \%$ & IRT/DNA & Luminex xTAG CF39 kit v2 & 39 & Always reported \\
\hline Washington & 86,577 & $2.2 \%$ & $\begin{array}{l}\text { IRT/IRT/ } \\
\text { DNA }^{d}\end{array}$ & p.Phe508del & 1 & N/A \\
\hline West Virginia & 20,825 & $0.5 \%$ & IRT/DNA & Hologic CF InPlex card & 41 & Always reported \\
\hline Wisconsin & 66,649 & $1.7 \%$ & IRT/DNA & Hologic InPlex CF molecular test & 23 & Not reported \\
\hline Wyoming & 7,644 & $0.2 \%$ & IRT/IRT/DNA & Luminex xTAG CF39 kit v2 & 39 & Reflex test, reported with p.R117H \\
\hline
\end{tabular}

IRT, immunoreactive trypsinogen; N/A, not applicable.

aThe number of variants tested does not include the p.I148T polymorphism or variants that are typically reflex-tested, such as p.F508C, p.I507V, p.I506V, and 3199del6. Some states have opted to drop the p.D1270N variant from the Hologic CF InPlex Card; as a result, they are testing for only 40 of 41 variants. bPolypyrimidine tract variants in intron 8 (IVS8; intron 9, IVS9 in sequential nomenclature). clowa, North Dakota, and South Dakota report the p.R117H variant only if 5 T is also present. ${ }^{\mathrm{d}}$ Montana and Washington perform limited DNA testing of low-birth-weight/premature infants. 
Molecular Test and the CF InPlex Card (Hologic, Bedford, MA) and the xTAG Cystic Fibrosis 39 Kit v2 (Luminex, Austin, TX). Of the 43 programs performing DNA testing, 19 (44.2\%; 19/51 $=37.3 \%$ overall) use the Hologic CF InPlex Card, 14 (32.6\%; $14 / 51=27.5 \%$ overall) offer the Luminex xTAG CF39 Kit v2, and four $(9.3 \% ; 4 / 51=7.8 \%$ overall $)$ apply the Hologic InPlex CF Molecular Test. In addition, two programs $(4.7 \% ; 2 / 51$ $=3.9 \%$ overall) test only the most common CFTR mutation, p.Phe508del (delF508 by legacy nomenclature), which accounts for $\sim 66 \%$ of identified mutant alleles worldwide. ${ }^{14}$ The remaining four programs $(9.3 \% ; 4 / 51=7.8 \%$ overall), grouped together in the "Other" category, use two-tier DNA testing that combines two separate DNA testing methods (California, Hawaii, and Nebraska) and/or a less common testing panel (California, Hawaii, and Utah). Regardless of the laboratory testing algorithm ultimately selected, CFNBS programs refer their screening-positive newborns to CF centers for sweat chloride testing, further clinical evaluation, and additional molecular testing if clinically indicated.

In 2001, the American College of Medical Genetics (ACMG, now the American College of Medical Genetics and Genomics) recommended a panel of 25 CFTR variants designed specifically for CF carrier screening in the general population; the panel was revised to 23 variants in $2004 .{ }^{4,18}$ Contrary to its original intended purpose, use of this panel has been extended to $\mathrm{CF}$ diagnostic testing and CFNBS. Not surprisingly, the 23 ACMGrecommended variants have also been incorporated into the three most common commercial panels used for CFNBS (Table 1). The composition of the Hologic InPlex CF Molecular Test, a US Food and Drug Administration-cleared genotyping test used in the CFNBS programs of four states, corresponds exactly to the ACMG panel. Expanded panels ranging from 32 to 97 variants, including the Hologic CF InPlex Card and the Luminex xTAG CF39 Kit v2, are used in 37 of $43(86.0 \%)$ CFNBS programs with a molecular tier $(37 / 51=72.5 \%$ overall $)$ (Table 2). These panels all contain the ACMG-recommended variants with one exception: the CFNBS program in California includes only 15 of the $23 \mathrm{ACMG}$ variants in favor of a modified panel that better reflects the CFTR alleles most frequently identified in CF patients in that state. ${ }^{19}$

Each of the four most common molecular CFNBS components (p.Phe508del testing, the InPlex CF Molecular Test, the CF InPlex Card, and the xTAG CF39 Kit v2) was evaluated to assess the percentages of individual ethnic ancestry groups in the "current" $2013 \mathrm{CF}$ population who would not be identified (Table 3, shaded rows). As expected, of the four methodologies, testing solely for p.Phe508del results in the highest percentage of CF patients with no detected CFTR variants, ranging from $10 \%$ of whites to $40 \%$ of Asians. The InPlex CF Molecular Test (comprising the 23 ACMG-recommended variants for carrier screening) reduced the number of CF individuals with two unidentified variants across all groups; however, individuals of Hispanic (16\%), black (18\%), Asian (28\%), and Native American (8\%) backgrounds were more likely to carry no ACMG variants when compared with whites $(3 \%)(P \leq 0.0001$

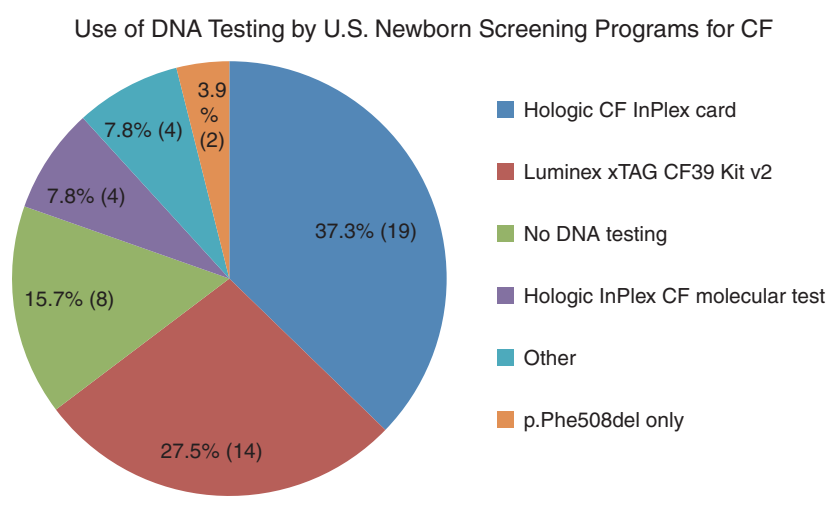

Figure 1 Use of a DNA testing component in cystic fibrosis newborn screening (CFNBS) testing algorithms is summarized for all 50 states and the District of Columbia in the United States ( $N=51$ programs). In the eight states (15.7\%) that do not currently include DNA testing as part of their CFNBS, two elevated IRT level results are used to consider a newborn screen-positive. The remaining states $(84.3 \%)$ incorporate DNA testing into their algorithms. In the context of CFNBS, three commercially available panels are frequently used. The "Other" category represents states that utilize twotier DNA testing, which combines two separate DNA testing methods, and/ or use a less common molecular panel.

for Hispanic, black, and Asian patients and $P=0.0003$ for Native Americans). Use of the 41-variant CF InPlex Card did not significantly lower the percentage of individuals with zero detected variants when compared with the 23-variant InPlex CF Molecular Test. Implementation of the 39-variant xTAG Cystic Fibrosis panel, however, resulted in modest, yet statistically significant $(P \leq 0.001)$, decreases by $3 \%$ in Hispanics and blacks each, for whom no variants are detected, as compared with the InPlex CF Molecular Test. The xTAG CF39 panel is also predicted to identify a two-allele genotype in a higher percentage of Hispanic and black individuals with CF (52 and 46\%, respectively) versus the InPlex CF Molecular Test (48 and 37\%, respectively; $P \leq 0.001$ ). With all panels, Native American individuals were closest in percentage to whites.

\section{DISCUSSION}

Delays in making a clinical and molecular diagnosis of CF are likely to negatively impact the health of an affected child and the well-being of his or her family. ${ }^{10,11,20,21}$ With a median diagnostic age of 2 to 4 weeks, CFNBS programs have markedly reduced the overall time to diagnosis; ${ }^{13}$ however, to make that benefit inclusive of all participating newborns, programs must carefully consider the composition of their testing algorithms. In the United States, the selection of CFNBS algorithms is made by individual states and the District of Columbia based on prioritization decisions and allotment of resources. Every program utilizes the IRT enzyme test, which is performed on dried blood spots derived from newborn heel sticks, as the primary triaging test. In children with an elevated result, however, this initial step is followed by a second IRT test, molecular testing for one or more CFTR variants, or both (Table 2 and Figure 1).

Every laboratory test has strengths and limitations. The IRT test, which is highly sensitive overall, can occasionally produce 
Table 3 Number of variants detected by ethnic ancestry and screening test in the genotyped CF population of 2013

\begin{tabular}{|c|c|c|c|c|c|c|c|c|c|c|}
\hline \multirow{2}{*}{$\begin{array}{l}\text { p.Phe508del } \\
\text { Variants detected }\end{array}$} & \multicolumn{2}{|c|}{ White only } & \multicolumn{2}{|c|}{ Hispanic only } & \multicolumn{2}{|c|}{ Black-any } & \multicolumn{2}{|c|}{ Asian-any } & \multicolumn{2}{|c|}{ Native-any } \\
\hline & $N$ & $\%$ & $N$ & $\%$ & $N$ & $\%$ & $N$ & $\%$ & $N$ & $\%$ \\
\hline 2 & 11,133 & 50 & 532 & 27 & 234 & 19 & 33 & 21 & 82 & 48 \\
\hline 1 & 8,775 & 40 & 835 & 43 & 522 & 43 & 60 & 38 & 60 & 35 \\
\hline 0 & 2,298 & 10 & 588 & 30 & 458 & 38 & 63 & 40 & 29 & 17 \\
\hline Total & 22,206 & $100 \%$ & 1,955 & $100 \%$ & 1,214 & $100 \%$ & 156 & $\sim 100 \%$ & 171 & $100 \%$ \\
\hline$P$ value ${ }^{a}$ & \multicolumn{2}{|c|}{ Reference group } & \multicolumn{2}{|c|}{$<0.0001$} & \multicolumn{2}{|c|}{$<0.0001$} & \multicolumn{2}{|c|}{$<0.0001$} & \multicolumn{2}{|c|}{0.005} \\
\hline Hologic InPlex CF molecular test $t^{b}$ & \multicolumn{2}{|c|}{ White only } & \multicolumn{2}{|c|}{ Hispanic only } & \multicolumn{2}{|c|}{ Black-any } & \multicolumn{2}{|c|}{ Asian-any } & \multicolumn{2}{|c|}{ Native-any } \\
\hline Variants detected & $N$ & $\%$ & $N$ & $\%$ & $N$ & $\%$ & $N$ & $\%$ & $N$ & $\%$ \\
\hline 2 & 16,782 & 76 & 933 & 48 & 454 & 37 & 48 & 31 & 116 & 68 \\
\hline 1 & 4,775 & 22 & 712 & 36 & 536 & 44 & 64 & 41 & 42 & 25 \\
\hline 0 & 649 & 3 & 310 & 16 & 224 & 18 & 44 & 28 & 13 & 8 \\
\hline Total & 22,206 & $\sim 100 \%$ & 1,955 & $100 \%$ & 1,214 & $\sim 100 \%$ & 156 & $100 \%$ & 171 & $\sim 100 \%$ \\
\hline$P$ value & \multicolumn{2}{|c|}{ Reference group } & \multicolumn{2}{|c|}{$<0.0001$} & \multicolumn{2}{|c|}{$<0.0001$} & \multicolumn{2}{|c|}{$<0.0001$} & \multicolumn{2}{|c|}{0.0003} \\
\hline Hologic CF InPlex card & \multicolumn{2}{|c|}{ White only } & \multicolumn{2}{|c|}{ Hispanic only } & \multicolumn{2}{|c|}{ Black-any } & \multicolumn{2}{|c|}{ Asian-any } & \multicolumn{2}{|c|}{ Native-any } \\
\hline Variants detected & $N$ & $\%$ & $N$ & $\%$ & $N$ & $\%$ & $N$ & $\%$ & $N$ & $\%$ \\
\hline 2 & 17,082 & 77 & 997 & 51 & 477 & 39 & 53 & 34 & 121 & 71 \\
\hline 1 & 4,502 & 20 & 678 & 35 & 528 & 43 & 61 & 39 & 38 & 22 \\
\hline 0 & 622 & 3 & 280 & 14 & 209 & 17 & 42 & 27 & 12 & 7 \\
\hline Total & 22,206 & $100 \%$ & 1,955 & $100 \%$ & 1,214 & $\sim 100 \%$ & 156 & $100 \%$ & 171 & $100 \%$ \\
\hline$P$ value ${ }^{a}$ & \multicolumn{2}{|c|}{ Reference group } & \multicolumn{2}{|c|}{$<0.0001$} & \multicolumn{2}{|c|}{$<0.0001$} & \multicolumn{2}{|c|}{$<0.0001$} & & 001 \\
\hline Luminex xTAG CF39 kit v2 & Whi & & Hispa & c only & Bla & any & & -any & Nat & e-any \\
\hline Variants detected & $N$ & $\%$ & $N$ & $\%$ & $N$ & $\%$ & $N$ & $\%$ & $N$ & $\%$ \\
\hline 2 & 17,195 & 77 & 1,024 & 52 & 554 & 46 & 57 & 37 & 121 & 71 \\
\hline 1 & 4,433 & 20 & 682 & 35 & 481 & 40 & 63 & 40 & 38 & 22 \\
\hline 0 & 578 & 3 & 249 & 13 & 179 & 15 & 36 & 23 & 12 & 7 \\
\hline Total & 22,206 & $100 \%$ & 1,955 & $100 \%$ & 1,214 & $\sim 100 \%$ & 156 & $100 \%$ & 171 & $100 \%$ \\
\hline$P$ value $^{\mathrm{a}}$ & Refere & oup & $<0$ & & & & & 001 & & 003 \\
\hline
\end{tabular}

Percentages are rounded and may not add up to exactly $100 \%$, indicated, where applicable, by " $100 \%$." Shaded rows indicate the percentages of each ethnic ancestry group that would not be identified by the given testing method.

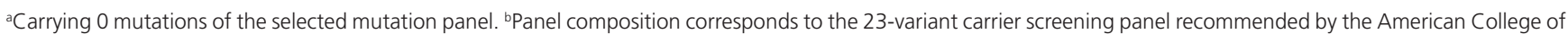
Medical Genetics and Genomics (ACMG).

false-negative results in the presence of meconium ileus. As is typical for a screening test, CFNBS emphasizes sensitivity more than specificity; hence, false-positive results are common and expected. Algorithms based on single (IRT) or sequential (IRT+IRT) hypertrypsinogenemia testing have a reported sensitivity of $85-90 \%$, with a considerable proportion $(\sim 94 \%)$ of false-positive results for single-tier IRT tests. ${ }^{12,19,22,23}$ These can result from, for example, clinical conditions, such as perinatal stress, low Apgar scores, and premature birth, as well as ethnic background, such as African ancestry. ${ }^{24}$ Given that IRT levels in infants without CF decrease with age, a follow-up IRT test, as applied in 18 of 51 programs in the United States (35.3\%), improves specificity (Table 2 ). ${ }^{8}$ Of these 18 programs, 10 also incorporate molecular testing but 8 do not. When a CFNBS algorithm does not include a molecular component, however, the opportunities for clinical and etiologic confirmation, prognostic assessment, and specific genetic counseling are missed, or at the least postponed.

In 43 of 51 programs (84.3\%), IRT testing is combined with DNA analysis in the following ways: IRT+DNA,
IRT+IRT+DNA, IRT+DNA+DNA, or IRT+IRT+DNA+DNA (Table 2). In such programs, the newborn is referred for clinical evaluation if one or more CFTR variants are identified. Whereas enhanced specificity is intrinsic to a DNA testing component, one added advantage of combining IRT testing with DNA analysis is enhanced screening program sensitivity because a larger proportion of IRT-positive newborns (1.0-5.0 vs. $0.5-1.0 \%$ ) is typically identified for second-tier testing. ${ }^{25-27}$ Although this approach may result in a larger number of CF center referrals based on infants with an initial elevated IRT level and who carry a single CFTR variant, cost-effectiveness may prevail because of reductions in morbidity and hospitalizations of successfully identified patients. ${ }^{28}$ To address loss of sensitivity due to a limited number of CFTR mutations on panels, many programs have implemented a "fail-safe" IRT procedure in which newborns with a very high IRT value (typically, at or above the 99.8th percentile) and no identified mutations are automatically referred for clinical evaluation. ${ }^{26,27}$ However, such an approach is nonspecific and associated with disadvantages such as a very low positive predictive value $(<1 \%) .{ }^{29,30}$ 
Clinical referral of infants who do not have CF can be further limited by including a second level of DNA testing. In California, for example, newborns with a single variant on the initial panel test receive sequencing of the CFTR gene and are referred to a CF center only when an additional reportable variant is identified. A screening approach that includes comprehensive sequence analysis, even though it cannot detect up to $2.4 \%$ of CF cases with gross deletions and insertions, ${ }^{16}$ is expected to miss the smallest number of patients, given that some infants (at least $6 \%$ of cases) have inconclusive initial sweat chloride levels and symptoms that cannot solidify a clinical diagnosis of CF in the first months of life. ${ }^{19,28,31}$

In the ethnically diverse population of the United States, the selection of variants for molecular panel testing warrants special consideration. Splice-site variant 3120+1G>A (c.2988+1G>A) illustrates this well: with a prevalence of $10-12 \%$, this variant is the second most common variant identified in black individuals with $\mathrm{CF}$, although it is relatively rare in non-Hispanic whites. ${ }^{18,32}$ This variant, as one of the 23 ACMG-recommended carrier screening variants, is included in most molecular CFNBS components (Table 1). In our original study of 3,496 nonwhite and 22,206 white CF patients, both the frequency and the spectrum of nonwhite CFTR variants were significantly different in comparison with whites. ${ }^{16}$ When the 23 -variant carrier screening panel, which is frequently selected for diagnostic testing, was assessed for its ability to identify variants across ethnic ancestry groups, CF patients of Hispanic, black, or Asian ancestry were less likely to carry two variants and more likely to have no detected CFTR variants $(P \leq 0.0001)$. Although similar results were obtained for Native American patients, this group appeared to be the most genetically similar to the white population, probably because of historic admixture. These findings confirmed that the ACMG list of 23 variants that was specifically developed for population carrier screening is inadequate as a general diagnostic $\mathrm{CF}$ test. Although expanded variant panels are commercially available and claim to be enriched for variants with increased prevalence in nonwhite populations, these panels were typically designed before the mutation spectrum of nonwhite groups had been extensively studied. Therefore, such panels have inherently limited value for diverse populations in the context of carrier screening, diagnostic testing, and CFNBS. ${ }^{16,33}$

To minimize disparities resulting from population differences in the clinical sensitivity of neonatal screening algorithms, racial/ethnic CFTR variant diversity should be considered. This point is illustrated by a variant detection comparison of the panels most commonly used for CFNBS: the Hologic InPlex CF Molecular Test that reflects the 23 ACMG variants, the Hologic CF InPlex Card with these 23 variants plus 18 additional variants evaluated in this study, and the Luminex xTAG CF39 assay with the 23 variants plus 16 additional variants included in this investigation (Tables 1 and 2). These panels, together with the approach of evaluating only for p.Phe508del, account for 93.0\% (40/43) of targeted variant test components in CFNBS algorithms. Each of these modalities, however, would detect significantly fewer variants in Hispanic, black, and Asian newborns as compared with whites $(P \leq 0.0001)$. A significant decrease in detected variants would also be observed in Native Americans ( $P$ values ranging from 0.001 to 0.0003 ), but of all the nonwhite groups, Native Americans would be closest in percentage to whites (Table 3). Because the panels are composed differently (Table 1), their detection rates in various populations are not the same. When compared with the 23-variant Hologic InPlex CF Molecular Test, the Hologic CF InPlex Card assay demonstrated improved detection for Hispanic newborns expected to be identified with two variants $(P=0.004)$, whereas the Luminex xTAG CF39 assay demonstrated an advantage for the percentages of affected Hispanic and black newborns $(P \leq$ 0.0001 each).

In addition, small differences between panels are observed in the percentages of affected nonwhite newborns who would be missed altogether because they had no variants identified. Among the three panels, these percentages range from 13 to $16 \%$ for Hispanics, 15 to $18 \%$ for blacks, 23 to $28 \%$ for Asians, and 7 to $8 \%$ for Native American newborns. Whereas the differences within each group are minor, an appreciable proportion of children with $\mathrm{CF}$ in nonwhite populations would be missed, as opposed to $3 \%$ of babies with white-only ancestry. Aside from the disproportionate identification of affected white versus nonwhite newborns inherent in most CFNBS algorithms because of panel variant selection, differences in the frequency of various types of mutations also exist. Whereas the majority of $\mathrm{CF}$ alleles in nonwhite patients affects single nucleotides, gene rearrangements by deletions and duplications account for up to $17 \%$ of unidentified $\mathrm{CF}$ alleles after sequencing. ${ }^{16}$ As documented in the CF Foundation Patient Registry, CFTR gene rearrangements occur relatively frequently in nonwhite CF patients, with Asians appearing to have the highest overall frequency, accounting for $2.4 \%$ of all identified alleles. ${ }^{16}$

Given these findings, what would be a reasonable strategy to minimize disparities? The optimal CFNBS design would include appropriate variants that fully reflect the spectrum of ethnicities in each region. For example, expanded variant panels such as the Hologic CF InPlex Card and the Luminex xTAG CF39 assay provide more comprehensive genotyping (i.e., detection of two variants) for Hispanics and blacks than a smaller panel based on the 23 ACMG variants. Therefore, larger CFNBS panels may result in more urgent patient referral and earlier care for Hispanic and black patients based on two identified variants on the NBS report, potentially narrowing disparities in the health of nonwhites versus whites with CF. However, the use of panels is inherently inequitable and can be expected to limit parity in any state and to particularly affect programs that use a standard panel and serve a nonhomogeneous population. To improve the current algorithms, especially in states with ethnic diversity, several options could be considered: (i) DNA from IRT-positive newborns with known nonwhite ancestry could be sequenced, instead of tested by a panel of variants; (ii) DNA from IRT-positive newborns with known nonwhite ancestry could be tested with a variant panel 
that is more inclusive of the constituency of a particular state or program, followed by a sequence-analysis step in the case of one identified variant; and (iii) DNA from all IRT-positive newborns could be sequenced instead of tested by a panel. With the rapid adoption of next-generation sequencing in various clinical settings, which overall can be more efficient and economical than Sanger sequencing, ${ }^{34,35}$ these options may be within reach for CFNBS. ${ }^{36-40}$ Each of these scenarios would need to be carefully weighed and, if adopted, monitored for sensitivity, specificity, cost, and anticipated outcomes.

At present, every program must conduct its own cost-benefit analysis regarding feasibility and overall practicality of offering inclusive molecular CFNBS testing of hypertrypsinogenemic infants. Inevitably, screening programs will fail to detect some individuals in order to be cost-effective. With careful evaluation of available algorithms and regional demographics, however, desirable sensitivities could be achieved for all populations and the differences between racial/ethnic groups could be minimized with the vital benefit of avoiding disproportionate disease burdens. In CF, a condition with considerable allelic heterogeneity and more than 2,000 CFTR variants described across populations (http://www.genet.sickkids.on.ca/ $\mathrm{cftr} /$ StatisticsPage.html), a more comprehensive approach may well make sense.

In conclusion, this is the first large-scale and in-depth evaluation of the impact of CFNBS algorithm selection on nonwhite newborns with CF in the United States. Longitudinal analysis of detection rates and outcome data, together with the implementation of refined algorithms as new approaches become available, can facilitate optimization of CFNBS and benefit affected newborns, their families, and society at large.

\section{ACKNOWLEDGMENTS}

This study was generously supported by a clinical research grant from Cystic Fibrosis Foundation Therapeutics and by the CF Foundation, which provided access to data in the CF Foundation Registry. We are grateful for the cooperation of the CF centers with nonwhite patients and indebted to the participating CF patients, without whom progress would not be possible.

\section{DISCLOSURE}

The authors declare no conflict of interest.

\section{REFERENCES}

1. Welsh MJ, Ramsey BW, Accurso F, Cutting GR. Cystic fibrosis. In: Valle D, Beaudet AL, Vogelstein B, et al (eds). OMMBID-The Online Metabolic and Molecular Bases of Inherited Diseases.McGraw-Hill: New York, 2013. http:// ommbid. mhmedical. com/content. aspx?bookid=474\&Sectionid=45374215. Accessed 25 August 2015

2. Farrell PM, Rosenstein BJ, White TB, et al.; Cystic Fibrosis Foundation. Guidelines for diagnosis of cystic fibrosis in newborns through older adults: Cystic Fibrosis Foundation consensus report. J Pediatr 2008;153:S4-S14.

3. Abeliovich $D$, Lavon $I P$, Lerer $I$, et al. Screening for five mutations detects $97 \%$ of cystic fibrosis (CF) chromosomes and predicts a carrier frequency of 1:29 in the Jewish Ashkenazi population. Am J Hum Genet 1992;51:951-956.

4. Grody WW, Cutting GR, Klinger KW, Richards CS, Watson MS, Desnick RJ; Subcommittee on Cystic Fibrosis Screening, Accreditation of Genetic Services Committee, ACMG. American College of Medical Genetics. Laboratory standards and guidelines for population-based cystic fibrosis carrier screening. Genet Med 2001:3:149-154.

5. Palomaki GE, FitzSimmons SC, Haddow JE. Clinical sensitivity of prenatal screening for cystic fibrosis via CFTR carrier testing in a United States panethnic population. Genet Med 2004;6:405-414.

6. Hamosh A, FitzSimmons SC, Macek M Jr, Knowles MR, Rosenstein BJ, Cutting GR. Comparison of the clinical manifestations of cystic fibrosis in black and white patients. J Pediatr 1998;132:255-259.

7. Rosenstein BJ, Cutting GR. The diagnosis of cystic fibrosis: a consensus statement. Cystic Fibrosis Foundation Consensus Panel. J Pediatr 1998;132:589-595.

8. Grosse SD, Boyle CA, Botkin JR, et al.; CDC. Newborn screening for cystic fibrosis: evaluation of benefits and risks and recommendations for state newborn screening programs. MMWR Recomm Rep 2004;53:1-36.

9. Spencer DA, Venkataraman M, Weller PH. Delayed diagnosis of cystic fibrosis in children from ethnic minorities. Lancet 1993;342:238.

10. Accurso FJ, Sontag MK, Wagener JS. Complications associated with symptomatic diagnosis in infants with cystic fibrosis. J Pediatr 2005;147(suppl 3):S37-S41.

11. Campbell PW 3rd, White TB. Newborn screening for cystic fibrosis: an opportunity to improve care and outcomes. J Pediatr 2005;147(suppl 3):S2-S5.

12. Wagener JS, Zemanick ET, Sontag MK. Newborn screening for cystic fibrosis. Curr Opin Pediatr 2012;24:329-335.

13. Sanders DB, Lai HJ, Rock MJ, Farrell PM. Comparing age of cystic fibrosis diagnosis and treatment initiation after newborn screening with two common strategies. J Cyst Fibros 2012;11:150-153.

14. Bobadilla JL, Macek M Jr, Fine JP, Farrell PM. Cystic fibrosis: a worldwide analysis of CFTR mutations-correlation with incidence data and application to screening. Hum Mutat 2002;19:575-606.

15. Alper OM, Wong $L$, Young $S$, et al. Identification of novel and rare mutations in California Hispanic and African American cystic fibrosis patients. Hum Mutat 2004;24:353.

16. Schrijver I, Pique L, Graham S, Pearl M, Cherry A, Kharrazi M. The spectrum of CFTR variants in nonwhite cystic fibrosis patients: implications for molecular diagnostic testing. J Mol Diagn 2016;18:39-50.

17. Martin JA, Hamilton BE, Osterman MJ, Curtin SC, Matthews TJ. Births: final data for 2013. Nat/ Vital Stat Rep 2015;64:1-65.

18. Watson MS, Cutting GR, Desnick RJ, et al. Cystic fibrosis population carrier screening: 2004 revision of American College of Medical Genetics mutation panel. Genet Med 2004;6:387-391.

19. Prach L, Koepke R, Kharrazi M, et al.; California Cystic Fibrosis Newborn Screening Consortium. Novel CFTR variants identified during the first 3 years of cystic fibrosis newborn screening in California. J Mol Diagn 2013;15:710-722.

20. Farrell PM, Lai HJ, Li Z, et al. Evidence on improved outcomes with early diagnosis of cystic fibrosis through neonatal screening: enough is enough! J Pediatr 2005;147(suppl 3):S30-S36.

21. Kharrazi M, Kharrazi LD. Delayed diagnosis of cystic fibrosis and the family perspective. J Pediatr 2005;147(suppl 3):S21-S25.

22. Farrell MH, Farrell PM. Newborn screening for cystic fibrosis: ensuring more good than harm. J Pediatr 2003;143:707-712.

23. Padoan R, Genoni S, Moretti E, Seia M, Giunta A, Corbetta C. Genetic and clinical features of false-negative infants in a neonatal screening programme for cystic fibrosis. Acta Paediatr 2002;91:82-87.

24. Rock MJ, Mischler EH, Farrell PM, Bruns WT, Hassemer DJ, Laessig RH. Immunoreactive trypsinogen screening for cystic fibrosis: characterization of infants with a false-positive screening test. Pediatr Pulmonol 1989;6:42-48.

25. Narzi L, Lucarelli M, Lelli A, et al. Comparison of two different protocols of neonatal screening for cystic fibrosis. Clin Genet 2002;62:245-249.

26. Comeau AM, Parad RB, Dorkin HL, et al. Population-based newborn screening for genetic disorders when multiple mutation DNA testing is incorporated: a cystic fibrosis newborn screening model demonstrating increased sensitivity but more carrier detections. Pediatrics 2004;113:1573-1581.

27. CLSI. Newborn Screening for Cystic Fibrosis; Approved Guideline. CLSI document I/LA35-A.Clinical and Laboratory Standards Insitute: Wayne, PA, 2011.

28. van den Akker-van Marle ME, Dankert HM, Verkerk PH, Dankert-Roelse JE. Cost-effectiveness of 4 neonatal screening strategies for cystic fibrosis. Pediatrics 2006;118:896-905

29. Giusti R, Badgwell A, Iglesias AD; New York State Cystic Fibrosis Newborn Screening Consortium. New York State cystic fibrosis consortium: the first 2.5 years of experience with cystic fibrosis newborn screening in an ethnically diverse population. Pediatrics 2007;119:e460-e467. 


\section{ORIGINAL RESEARCH ARTICLE}

30. Hale JE, Parad RB, Dorkin HL, et al. Cystic fibrosis newborn screening: using experience to optimize the screening algorithm. J Inherit Metab Dis 2010;33(suppl 2):S255-S261.

31. Kharrazi M, Yang J, Bishop T, et al.; California Cystic Fibrosis Newborn Screening Consortium. Newborn screening for cystic fibrosis in California. Pediatrics 2015;136:1062-1072.

32. Macek M Jr, Mackova A, Hamosh A, et al. Identification of common cystic fibrosis mutations in African-Americans with cystic fibrosis increases the detection rate to 75\%. Am J Hum Genet 1997;60:1122-1127.

33. Grody WW, Cutting GR, Watson MS. The cystic fibrosis mutation "arms race": when less is more. Genet Med 2007;9:739-744.

34. Schrijver I, Aziz N, Farkas DH, et al. Opportunities and challenges associated with clinical diagnostic genome sequencing: a report of the Association for Molecular Pathology. J Mol Diagn 2012;14:525-540.

35. Aziz N, Zhao Q, Bry L, et al. College of American Pathologists' laboratory standards for next-generation sequencing clinical tests. Arch Pathol Lab Med 2015;139:481-493.
36. Trujillano D, Ramos MD, González J, et al. Next generation diagnostics of cystic fibrosis and CFTR-related disorders by targeted multiplex high-coverage resequencing of CFTR. J Med Genet 2013;50: 455-462.

37. Abou Tayoun AN, Tunkey CD, Pugh TJ, et al. A comprehensive assay for CFTR mutational analysis using next-generation sequencing. Clin Chem 2013;59:1481-1488.

38. Grosu DS, Hague L, Chelliserry M, et al. Clinical investigational studies for validation of a next-generation sequencing in vitro diagnostic device for cystic fibrosis testing. Expert Rev Mol Diagn 2014;14:605-622.

39. Baker MW, Atkins AE, Cordovado SK, Hendrix M, Earley MC, Farrell PM. Improving newborn screening for cystic fibrosis using next-generation sequencing technology: a technical feasibility study. Genet Med 2016;18: 231-238.

40. Lefterova MI, Shen P, Odegaard Jl, et al. Next-generation molecular testing of newborn dried blood spots for cystic fibrosis. J Mol Diagn 2016;18: 267-282. 